\title{
Preparation of acetazolamide composite microparticles by supercritical anti-solvent techniques
}

\author{
Ana Rita C. Duarte ${ }^{\mathrm{a}, *}$, Christelle Roy ${ }^{\mathrm{b}}$, Arlette Vega-González ${ }^{\mathrm{b}}$, \\ Catarina M.M. Duarte ${ }^{\mathrm{a}}$, Pascale Subra-Paternault ${ }^{\mathrm{b}}$ \\ ${ }^{a}$ Nutraceuticals and Delivery Laboratory, ITQB/IBET, Aptd. 12, 2781-901 Oeiras, Portugal \\ ${ }^{\mathrm{b}}$ Laboratoire d'Ingénierie des Matériaux et des Hautes Pressions, C.N.R.S., Institut Galilée, Université Paris 13, \\ 99 Avenue Jean Baptiste Clément, 93430 Villetaneuse, France \\ Received 26 January 2006; received in revised form 22 September 2006; accepted 25 September 2006 \\ Available online 29 September 2006
}

\begin{abstract}
The possibility of preparation of ophthalmic drug delivery systems using compressed anti-solvent technology was evaluated. Eudragit RS 100 and RL 100 were used as drug carriers, acetazolamide was the model drug processed. Compressed anti-solvent experiments were carried out as a semi-continuous or a batch operation from a liquid solution of polymer(s) + solute dissolved in acetone. Both techniques allowed the recovery of composite particles, but the semi-continuous operation yielded smaller and less aggregated populations than the batch operation. The release behaviour of acetazolamide from the prepared microparticles was studied and most products exhibited a slower release than the single drug. Moreover, the release could be controlled to some extent by varying the ratio of the two Eudragit used in the formulation and by selecting one or the other anti-solvent technique. Simple diffusion models satisfactorily described the release profiles. Composites specifically produced by semi-continuous technique have a drug release rate controlled by a diffusion mechanism, whereas for composites produced by the batch operation, the polymer swelling also contributes to the overall transport mechanism.
\end{abstract}

(C) 2006 Elsevier B.V. All rights reserved.

Keywords: Acetazolamide; Eudragit; Supercritical fluids; SAS; GAS; Drug delivery

\section{Introduction}

Controlled drug delivery products using biocompatible or biodegradable polymers, have received considerable attention in the last years. In general, these substances provide a better controlled rate of assumption of the drug by the body improving its therapeutic action. In fact, there is a growing interest of the pharmaceutical industry in the development of these systems (Heller, 1996). Meanwhile, intensive world-wide research related to the application of high-pressure and supercritical fluids in the field of particle formation, encapsulation and impregnation of polymeric matrixes has proven the efficiency of SCF-technology to generate improved delivery systems (Reverchon, 2002; Jung and Perrut, 2001; Vemavarapu et al., 2005). Supercritical fluid

\footnotetext{
* Corresponding author. Fax: +3314940 3414.

E-mail addresses: rduarte@itqb.unl.pt (A.R.C. Duarte), subra@limhp.univ-paris13.fr (P. Subra-Paternault).
}

technology has became a very attractive alternative to the conventional processes due to the strict international regulations that rigorously control the presence of organic solvents in products for medical or pharmaceutical applications. This technology warrants a better removal of these solvents without exposing drugs to high temperatures which may degrade them (a dry solid product is easily obtained, just by manipulating pressure). Furthermore, supercritical carbon dioxide has major advantages when compared to most traditional solvents: it is non-toxic, non-flammable and not expensive. Carbon dioxide can also be recovered and reused, and therefore, do not contribute to the greenhouse effect. Because of all these advantages, supercritical processes are often referred and classified as "green" and "environmental friendly" processes (CPMP/ICH/283/95, 1997).

The $\mathrm{CO}_{2}$ anti-solvent technique is one of the techniques commonly used to prepare new drug delivery systems. The technique takes advantage of the anti-solvent effect of supercritical $\mathrm{CO}_{2}$ to precipitate the substrate(s) initially dissolved in a liquid solvent. When performed in a semi-continuous mode (SAS), it is possible 
to control the morphology, size and size distribution of the particles by manipulating process variables, such as temperature, pressure and flow rate (Reverchon and Adami, 2005; Subra and Jestin, 1999). In a batch mode (GAS, for gaseous anti-solvent), the compressed anti-solvent is added to a given volume of liquid solution until the final pressure is reached (Subra and Jestin, 1999). Although examples of drug delivery systems produced by batch are scare, this mode offers larger times for precipitation and/or interactions between drug and polymer than the semi-continuous operation, giving potential for formulations of different characteristics.

Controlled drug delivery occurs when a polymer/drug system is designed to release the drug in a predetermined manner. The main purpose of these release systems is to achieve a more effective therapy, that is, a delivery profile that would yield a high blood level of the drug over a long period of time, avoiding the large fluctuations in drug concentration and reducing the need of several administrations (Brannon-Peppas, 1997).

Glaucoma is a serious eye disease that can lead to irreversible blindness. Glaucoma is a group of ophthalmic disorders characterized by an increase in intraocular pressure, which results in damage to the optic nerve and visual field disturbances. Agents used to treat glaucoma are designed to decrease intraocular pressure. Nevertheless, these drugs present severe side effects therefore, there is an urgent need to develop new drug delivery systems. Various classes of drugs used in the treatment of glaucoma include, among others, carbonic anhydrase inhibitors (CAIs). Acetazolamide ( $N$-5-sulfamoyl-1,3,4-thiadiazol-2-yl)acetamide (Fig. 1) is a CAI and has been an integral part of anti-glaucoma treatment for more than 40 years (Kaur et al., 2002).

Modern sustained release dosage forms require excipients that can ensure a release rate of the active drug, which is reproducible in a narrow range. Eudragit ${ }^{\circledR}$ (Fig. 2) polymers fulfill these requirements to a very high extend and enable research and development of tailor-made solutions (Anon, 2006, http://www.roehm.de/). The Eudragit ${ }^{\circledR}$ grades for sustained release formulations are based on copolymers of acrylate and methacrylates with quaternary ammonium groups as functional groups as well as ethylacrylate methylmethacrylate copolymers with a neutral ester group. Eudragit ${ }^{\circledR}$ RS and RL are waterinsoluble, nevertheless they are both swellable, that is, permeable to water, representing thus interesting materials for the dispersion of drugs (Pignatello et al., 2003). This permeability is due to the quaternarium ammonium groups present in their

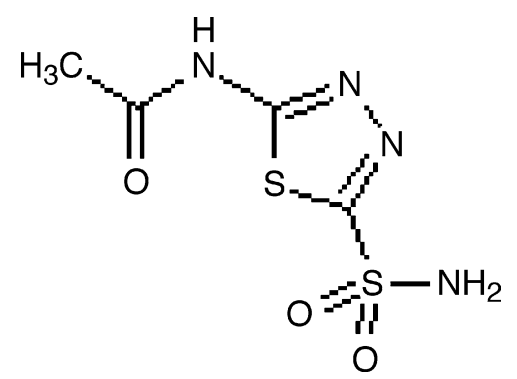

Fig. 1. Chemical structure of acetazolamide.

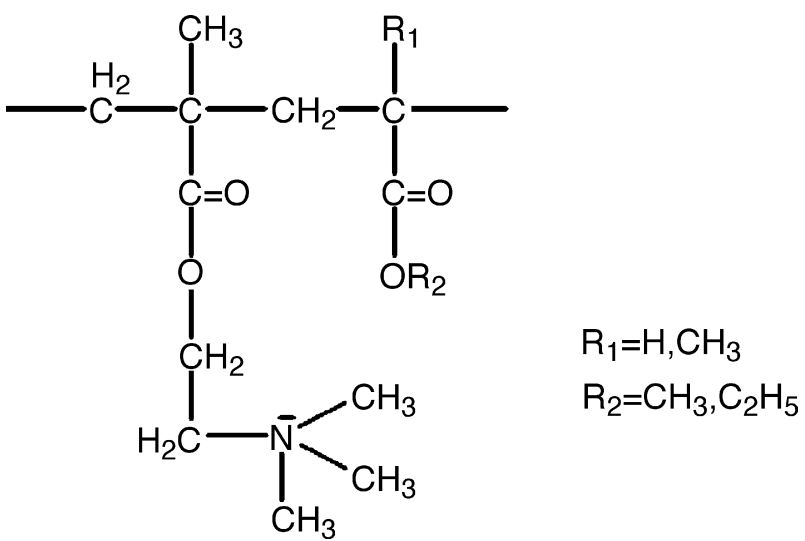

Fig. 2. Chemical structure of Eudragit ${ }^{\circledR}$.

structure. The Eudragit ${ }^{\circledR}$ RL-types are highly permeable while the Eudragit ${ }^{\circledR}$ RS-types are poorly permeable; therefore, release profiles can be varied by mixing RL and RS types in different ratios.

Nanoparticle coating and encapsulation of silica particles with Eudragit ${ }^{\circledR}$ has been successfully described by Wang et al. (2004). Furthermore, the preparation of Eudragit ${ }^{\circledR}$ microspheres containing acetazolamide using the solvent evaporation method was appraised by Haznedar and Dortunç (2004). In this study, the possibility of preparing ophthalmic drug delivery systems using supercritical anti-solvent technology was evaluated. Eudragit $^{\circledR}$ RS 100 and RL 100 were used as drug carriers and acetazolamide was the drug processed. The experiments were performed at different operational conditions and with different compositions of polymer.

\section{Experimental}

\subsection{Materials}

Eudragit RL $100^{\circledR}$ and RS $100^{\circledR}$ from Degussa were kindly supplied by Société de Produits Chemiques Industriels. Acetazolamide ( $N$-5-sulfamoyl-1,3,4-thiadiazol-2-yl)-acetamide, CAS [59-66-5] (99\% purity), was purchased from Sigma-Aldrich. Acetone CAS [67-64-1] (99.8\% purity) was purchased from Prolabo (France). $\mathrm{CO}_{2}$ (99.5\%, industrial grade) was obtained from Air Liquide (France). All chemicals were used without any further purification.

\subsection{Experimental procedure}

\subsubsection{Semi-continuous anti-solvent technique}

The experimental work was performed at a laboratory scale. The apparatus is schematically presented in Fig. 3. Experiments were performed with the standard procedure already described by Vega-González et al. (2004). The apparatus works in a semicontinuous co-current mode, in which the $\mathrm{CO}_{2}$ anti-solvent and the liquid solution are separately fed to the top of the precipitator and are continuously discharged from the bottom.

The spray chamber (2) consists of a high-pressure vessel (Autoclave Engineers), $5 \mathrm{~cm}$ i.d. $\times 25 \mathrm{~cm}$ long with sapphire 


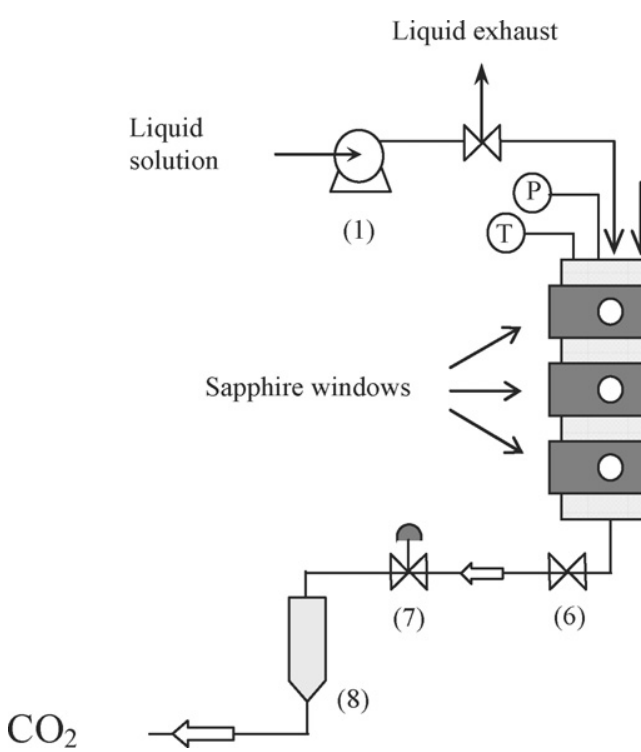

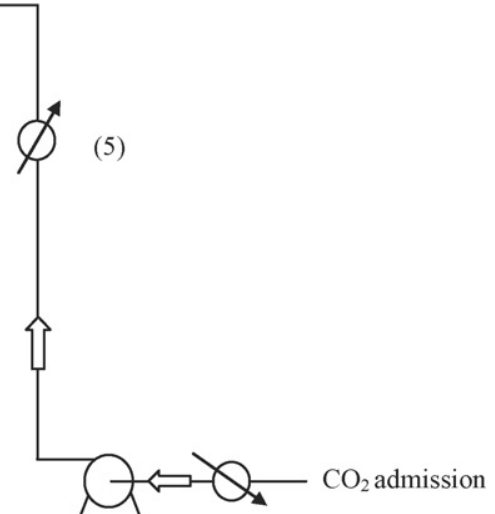

(4)
(3)

Fig. 3. Experimental apparatus: (1) liquid solution pump; (2) high-pressure vessel; (3) $\mathrm{CO}_{2}$ cooling bath; (4) $\mathrm{CO}_{2}$ pump; (5) $\mathrm{CO}_{2}$ heating bath; (6) valve; (7) micrometering valve; (8)separator.

windows at three different levels allowing a visual observation of both the spray and the precipitation. At the vessel bottom, the precipitated powder is collected onto a membrane filter placed on top of a stainless steel filter of $2 \mu \mathrm{m}$ porosity. The $\mathrm{CO}_{2}$ was cooled by flowing through a cold water + ethanol bath (3) and then was pumped at constant flow by using a reciprocating LEWA (EK3) pump (4). Prior to entering the precipitation chamber, it flowed through a second water bath (5) for an heating at the vessel temperature. The pressure inside the vessel was controlled downstream with a metering valve (7), and the temperature was controlled by heating jackets (Watlow). Acetone solutions of solutes were sprayed into the precipitation chamber by means of a reciprocating dual-piston minipump (Milton Roy LDC).

Once the temperature of the vessel had attained the desired value, the $\mathrm{CO}_{2}$ was pumped to the vessel until the desired pressure was reached; a constant flow was further maintained via the metering valve. The solution was introduced into the precipitation chamber through a spray-type nozzle of $100 \mu \mathrm{m}$, at a typical flow rate of $1.1 \mathrm{~mL} / \mathrm{min}$. After leaving the precipitator, the $\mathrm{CO}_{2}$ /acetone solution was depressurised across the metering valve and separated in a homemade cyclonic separator (8). Once the desired volume of solution was sprayed, fresh $\mathrm{CO}_{2}$ passed through the chamber in order to dry the precipitated powder. After approximately $40 \mathrm{~min}$ of purging with $\mathrm{CO}_{2}$, the vessel was slowly depressurized at the experimental temperature. Tubing and valves after the vessel were heated to prevent freezing due to $\mathrm{CO}_{2}$ expansion, and they were flushed with acetone between each experiment.

\subsubsection{Batch anti-solvent technique}

The set-up is presented schematically in Fig. 4. The apparatus and the procedure were previously described (De Gioannis et al., 2004).

Briefly, the crystalliser is a stainless steel vessel of $20 \mathrm{~cm}$ in height and $4.3 \mathrm{~cm}$ of internal diameter, with three sapphire win- dows to allow visual observations. The tank is equipped with four baffles that preclude the generation of a central vortex. The temperature is measured by a type $\mathrm{K}$ thermocouple with an accuracy of $1{ }^{\circ} \mathrm{C}$, and controlled by electric resistances. The pressure is measured by a pressure sensor with an accuracy of $0.1 \mathrm{MPa}$. The stirring is provided by a magnetically coupled stirrer with a eight-bladed Rushton-type turbine of $2.5 \mathrm{~cm}$ in diameter. The stirring rate was set at $505 \mathrm{rpm}$. The $\mathrm{CO}_{2}$, delivered by a high-pressure pump, is introduced into the solution through the turbine. At the vessel bottom, shut off and metering valves allow for discharging the solution and to regulate the

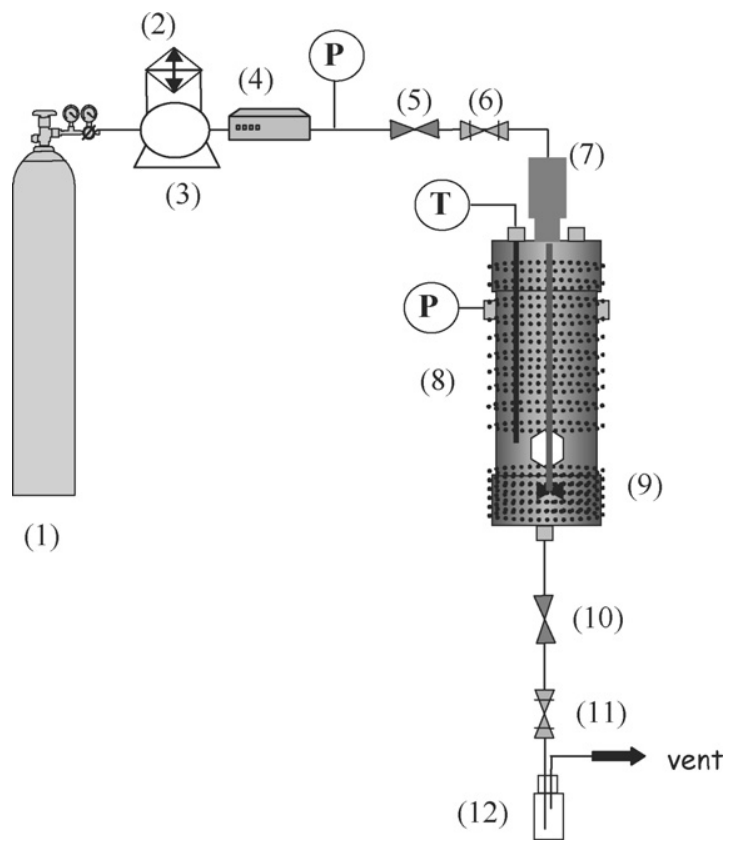

Fig. 4. (1) $\mathrm{CO}_{2}$ cylinder; (2) cooling unit; (3) $\mathrm{CO}_{2}$ pump; (4) flow meter; (5 and 10) valve; (6 and 11) metering valve; (7) stirrer; (8) crystalliser; (9) turbine; (12) solution recovery. 
crystalliser pressure during the final steps. A stainless steel frit of $5 \mu \mathrm{m}$ in porosity and a membrane of $0.22 \mu \mathrm{m}$ are located at the vessel bottom to collect the precipitated powder.

The procedure consisted in four steps, that are, (I) pressurization up to the desired final pressure by addition of $\mathrm{CO}_{2}$, (II) discharge of the solution, (III) crystals drying and (IV) depressurization. After the initial loading of the vessel with $65 \mathrm{~mL}$ of the polymer + drug solution and the obtaining of a stable temperature, $\mathrm{CO}_{2}$ was introduced by opening the inlet metering valve until that the vessel pressure equilibrated the $\mathrm{CO}_{2}$-cylinder pressure. The pump was further activated to continue the introduction up to the desired pressure. The $\mathrm{CO}_{2}$ feed was regulated around $3 \mathrm{bar} / \mathrm{min}$ (i.e. $12 \mathrm{~g} / \mathrm{min}$ ) for the experiments reported here, through the metering valve and the pump frequency. Upon the addition of $\mathrm{CO}_{2}$, the solvating ability of the newly formed mixture decreased and caused the solute precipitation. The discharge was carried out immediately after the pressurization step; the aim of the step is to wash out the $\mathrm{CO}_{2}+$ acetone mixture that contains the non-precipitated part of solute. The step was carried out by regulating the exit flow and compensating by fresh $\mathrm{CO}_{2}$, and lasted about $60 \mathrm{~min}$. The $\mathrm{CO}_{2}$ flow was further maintained during $45 \mathrm{~min}$ to dry the precipitates. Depressurization was finally performed by venting $\mathrm{CO}_{2}$ by the exit line. Crystals were collected for subsequent analysis; the ratio of the collected amount to the initial amount of components dissolved in the liquor defined the yield.

\subsubsection{Particle characterization}

2.2.3.1. Scaning electron microscopy-SEM. The morphology of polymer samples was analysed and imaged by scanning electron microscopy (SEM, Leica 5440) after sputter coating with gold-palladium to a thickness of approximately $90 \AA$.

2.2.3.2. Particle size and size distribution. The particle size and size distribution of the prepared microparticles were measured by Laser diffraction spectrometry (Coulter LS 130, Coulter Electronics). The dried powder samples were suspended in deionised water with a surfactant solution (Coulter Dispersant, Coulter) and sonicated for $1 \mathrm{~min}$ with an ultra-sound probe $(500 \mathrm{~W}$, Vibra Cell, Sonics \& Materials, Inc.) before measurement. The obtained homogeneous suspension was determined for the volume mean diameter, size distribution and polydispersity. Each sample was analysed three times.

2.2.3.3. In vitro drug release studies. Particles $(20 \mathrm{mg})$ were suspended in $40 \mathrm{~mL}$ of phosphate buffer solution ( $\mathrm{pH} 7.4)$ and simulated gastric fluid without pepsin ( $\mathrm{pH} 1.2)$ stirred at $150 \mathrm{rpm}$ at $37^{\circ} \mathrm{C} .0 .5 \mathrm{~mL}$ aliquots were withdrawn at predetermined time intervals and the same volume of fresh medium was added to the suspension. The samples were filtered $(0.45 \mu \mathrm{m}$, millipore filters) and analysed by HPLC (ThermoFinnigan Surveyor consisting of a quaternary pump, autosampler and diode array detector). A column (Merck Lichrospher $100 \mathrm{RP}-18 \mathrm{e}, 250 \mathrm{~mm} \times 4 \mathrm{~mm}$, $5 \mu \mathrm{m}$; cat. No. 1.50995) kept at room temperature, was used in isocratic conditions with a mobile phase consisting of sodium acetate trihydrated in water:methanol:acetonitrile (950:20:30), $\mathrm{pH}$ 4.0. The flow rate was $1.5 \mathrm{~mL} / \mathrm{min}$ and the volume of injec- tion was $20 \mu \mathrm{L}$. Quantitation was performed at $254 \mathrm{~nm}$ by external standard calibration. The total mass of released drug in each moment of the experiment was calculated taking into account the aliquots taken and the dilution produced by addition of fresh buffer.

\section{Results and discussion}

Preliminary experiments were carried out in order to precipitate pure polymers (Eudragit RS or RL) or a mixture of them in the ratio of 50:50, without acetazolamide. Attempts were not successful since the polymer created a film-like structure on the cylinder walls and did not precipitate as a powder. Nevertheless, Eudragit S100, which is an anionic polymer based on methacrylic acid esters has been precipitated using a similar apparatus to the one described from an acetone solution at 85 bar and $35^{\circ} \mathrm{C}$ (Fig. 5). The structural difference in these types of Eudragit is what probably causes the discrepancy in the results obtained.

This observed behaviour is in accordance with the few published results reporting precipitation of pure polymers or examples of encapsulation/coating with Eudragit assisted by supercritical techniques. Chattopadhyay and Gupta (2002) used Eudragit RS 100 for the production of magnetically responsive particles. Precipitation of the pure polymer have led to the formation of agglomerated spherical but flattened particles. The appearance was attributed to the decrease of the polymer glass transition temperature in the presence of supercritical $\mathrm{CO}_{2}$, which resulted in polymer softening. The main difference between their conditions and ours resided in the way of introducing the solution and $\mathrm{CO}_{2}$ into the vessel, and this might explain the different precipitation behaviour. The polymer swelling was exploited by Wang et al. (2004) for the purpose of coating silica with Eudragit RL 100 by supercritical anti-solvent technique. At the investigated conditions of 82.7 bar and $32.3^{\circ} \mathrm{C}$, it was assumed that the jet break-up generated droplets of polymer solution with entrapped nanoparticles, and that the subsequent crossing over the equilibrium boundary initiated the gelation of

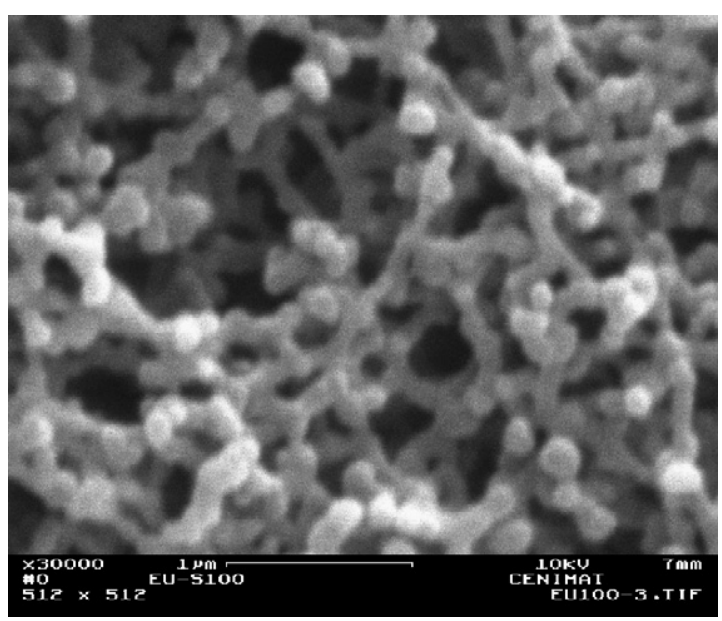

Fig. 5. SEM image of Eudragit S100 precipitated by SAS (semi-continuous mode). 
Table 1

Composites processed by the semi-continuous operation

\begin{tabular}{lllll}
\hline \#Run & Polymer & Drug & Yield $(\%)$ & Particle size $(\mu \mathrm{m})$ \\
\hline 1 & Eudragit RL 100 & ACTZ & 77 & 17.85 \\
2 & Eudragit RS 100 & ACTZ & 63 & 23.88 \\
3 & RL 100+RS 100 (50:50) & ACTZ & 66 & 21.32 \\
4 & RL 100+RS 100(70:30) & ACTZ & 70.7 & 19.76 \\
5 & RL 100+RS 100(30:70) & ACTZ & 74.8 & 17.04
\end{tabular}

Conditions: temperature of $37^{\circ} \mathrm{C}$, pressure of $83 \mathrm{bar}, \mathrm{CO}_{2}$ flow rate of $\sim 15 \mathrm{~mL} / \mathrm{min}$, solution flow rate $\sim 1.1 \mathrm{~mL} / \mathrm{min}$. ACTZ=acetazolamide; the reported particle size is the mean size of the distribution.

the polymer around the particles. Since the experimental conditions used in our study were $37^{\circ} \mathrm{C}$ and 83 bar, the obtained film-like structure can thus be explained by the swelling of the polymer at these conditions. Another type of Eudragit, Eudragit E 100 (tertiary ammonium groups) was processed in the presence of $\mathrm{CO}_{2}$ by the solution enhanced dispersion technique (SEDS) and no well-defined particles were obtained owing to its low Tg (Juppo et al., 2003). The combination of pressurized carbon dioxide with holt melt extrusion was also carried out (Verreck et al., in press), and it was again stated that $\mathrm{CO}_{2}$ acted as a plasticizer for Eudragit E 100.

\subsection{Precipitation of acetazolamide-loaded particles}

A first set of experiments was designed in the semicontinuous apparatus, to evaluate the effect of pressure, temperature and $\mathrm{CO}_{2}$ flow rate upon the recovery of powder; conditions were settled as proposed by Wang et al. (2004). The processed materials were a mixture of acetazolamide and one polymer. The results obtained were presented elsewhere (Vega-González et al., 2005). Briefly, the morphology of the particles were not strongly affected by the operational conditions, and we concluded that conditions to achieve a higher yield and smaller particle diameter in a semi-continuous operation were a temperature of $37^{\circ} \mathrm{C}$, a pressure of $83 \mathrm{bar}$ and a $\mathrm{CO}_{2}$ flow rate of $\sim 15 \mathrm{~mL} / \mathrm{min}$. The second set of experiments was then dedicated to test various formulations of drug and polymers. Conditions and results of experiments are summarized in Table 1 for the semi-continuous investigations. In these experiments, mixtures of Eudragit RS and RL (50:50; 70:30 and 30:70) were coprecipitated with acetazolamide. The drug concentration was constant and fixed at $\sim 8.2 \mathrm{mg} / \mathrm{mL}$ and the polymers:drug ratio was of $2: 1$ (w:w).

Particles produced by the semi-continuous mode were recovered as a powder made of spherical and elongated structures, as shown in Fig. 6. The composition of the polymer mixture does

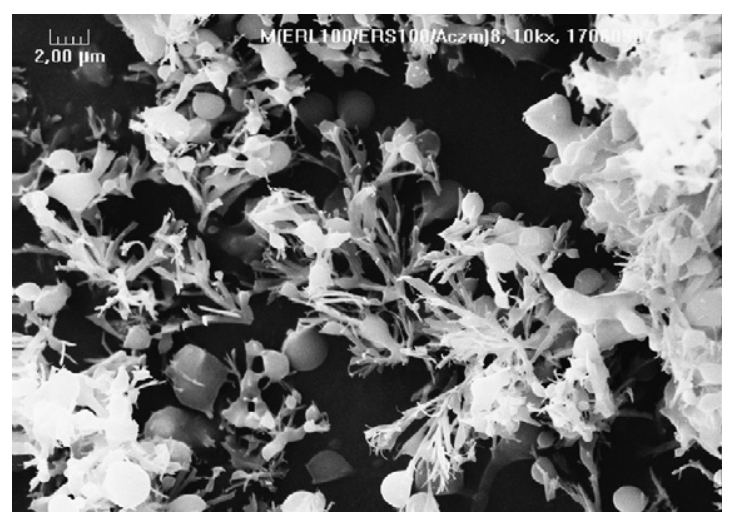

Fig. 6. SEM image of the precipitated acetazolamide + Eudragit blend (run \#4) by a semi-continuous operation.

not influence notably the yield or the mean particle size, since there is no straight tendency of those characteristics with the RL or RS content. However, it is worth noting that the presence of the drug allows for the precipitation of the polymer, whereas its processing as pure have led to a film deposition onto the vessel surface. Among the formulations proposed, the composition of 30:70 RL:RS was found to combine the smaller particle size and the higher yield. The batch operation was thus carried out with that mixture, keeping also the polymer:drug ratio to $2: 1$.

When the precipitation was carried out in the batch mode (Table 2), the recovered powder showed large agglomerates, made of rods embedded within a continuous film. The decrease of the operating temperature from 40 to $27^{\circ} \mathrm{C}$ and of the overall species concentration as well decreased the aggregation and the particle size range (run \#8, Fig. 7). Compared to the semicontinuous mode, the batch operation produced particles of larger size and more dispersed with a low yield in the $40 \%$ range; it is thus suggested that the semi-continuous mode is preferable for the precipitation of ACTZ composites.

\subsection{Release studies}

As the main goal of this work was to prepare a controlled release system for acetazolamide, the solubility of the pure drug was first evaluated in two biological fluids, that are, simulated gastric fluid ( $\mathrm{pH}$ 1.2) and PBS ( $\mathrm{pH} 7.4$ ) simulating the intestinal environment. In Fig. 8, the solubilization of acetazolamide as a function of time is presented for the two media.

As it can be observed, the pure drug is totally dissolved in the two media in $4 \mathrm{~h}$. However, acetazolamide solubilizes faster in a neutral environment than in the simulated gastric fluid and its complete dissolution is thus achieved in $1.5 \mathrm{~h}$ instead of $4 \mathrm{~h}$.

Table 2

Composites processed by the batch operation

\begin{tabular}{|c|c|c|c|c|c|}
\hline \#Run & Mixture & $C_{\mathrm{ACTZ}}(\mathrm{mg} / \mathrm{mL})$ & $T ; P_{\mathrm{f}}$ & Yield (\%) & Particle size $(\mu \mathrm{m})$ \\
\hline 6 & $\mathrm{ACTZ}+\mathrm{RS}+\mathrm{RL}$ & 8.2 & $40^{\circ} \mathrm{C} ; 90$ bar & 37.3 & $255-711$ \\
\hline 7 & $\mathrm{ACTZ}+\mathrm{RS}+\mathrm{RL}$ & 7.3 & $32^{\circ} \mathrm{C} ; 61$ bar & 45.7 & $117-498$ \\
\hline 8 & $\mathrm{ACTZ}+\mathrm{RS}+\mathrm{RL}$ & 4.9 & $27^{\circ} \mathrm{C} ; 57$ bar & 36.4 & $76-306$ \\
\hline
\end{tabular}

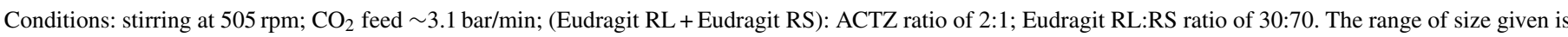
the D10 and D90 of the PSD. 

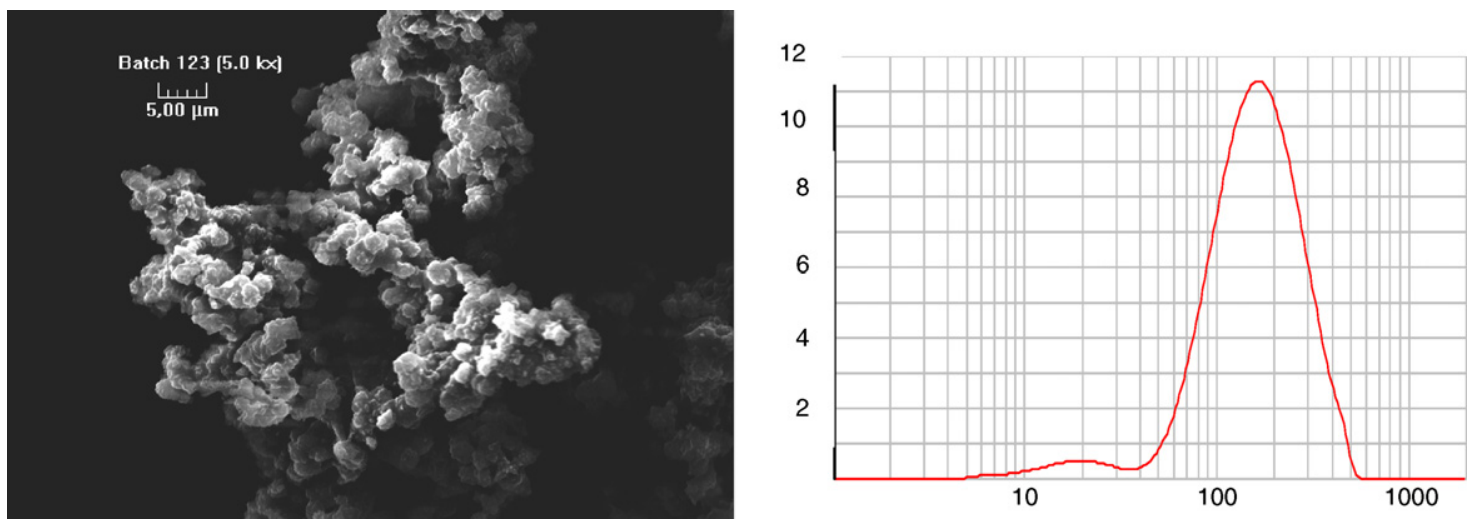

Fig. 7. SEM image and particle size distribution (vol.\%) of acetazolamide + Eudragit blend (run \#8) precipitated by batch anti-solvent operation.

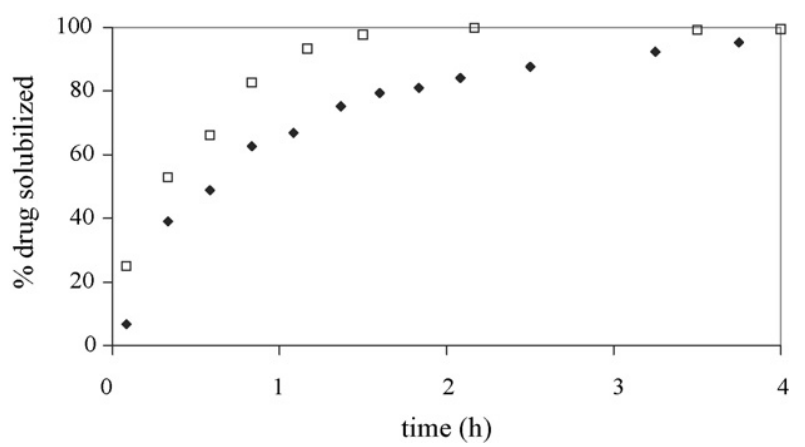

Fig. 8. Release profiles of the pure drug, acetazolamide $(\diamond) \mathrm{pH} 1.2$ and $(\square) \mathrm{pH}$ 7.4 .

This fast dissolution rate is clinically undesirable as it does not promote the contact of the drug with the mucosal membranes over a time sufficient enough to be absorbed, so the drug will be "washed out" of the body. Therefore a system that enables a sustained release of the drug is of great interest for the delivery of acetazolamide.

The comparison of the drug released from the microspheres prepared by semi-continuous operation and involving only one polymer (Eudragit RS or Eudragit RL) is presented Fig. 9. Although the two formulations induced a slower release of the drug, Eudragit RS 100 obviously ensured a more uniform release over a longer time than Eudragit RL 100.

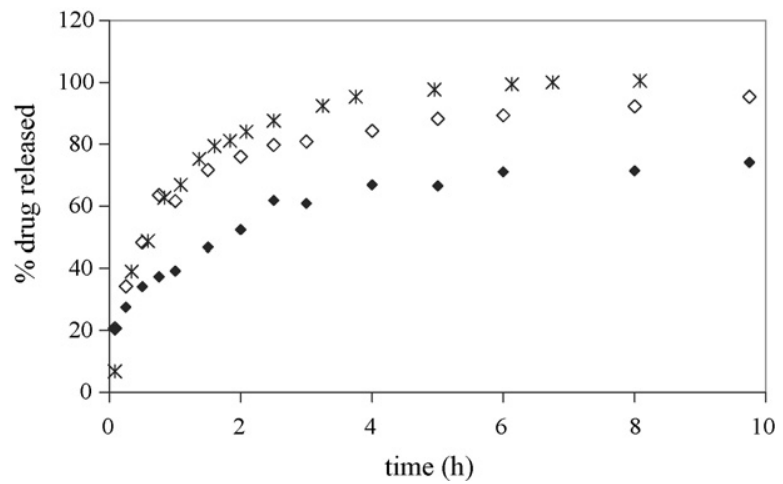

Fig. 9. Release profiles ( $\mathrm{pH} 1.2$ ) of the (*) pure drug and the systems $(\bullet)$ Eudragit RS 100 + acetazolamide (run \#2) and $(\diamond)$ Eudragit RL 100 + acetazolamide (run \#1).
The release profiles obtained for the two media, i.e. at $\mathrm{pH} 1.2$ and 7.4, exhibited the same behaviour than the pure drug, i.e., the release of the drug is faster in a neutral environment than in an acidic one (Fig. 10).

The release behaviour, either at $\mathrm{pH} 1.2$ or $\mathrm{pH} 7.4$, depends thus greatly on the type of polymer used in the formulation. Indeed, there is a large difference in the acetazolamide release from Eudragit RS 100 or from Eudragit RL 100, as already illustrated in Fig. 9. Acetazolamide release from Eudragit RS 100 miscrospheres was much slower and incomplete when compared to the spheres prepared with Eudragit RL 100 in which almost $80 \%$ of the drug is released after $3 \mathrm{~h}$. This discrepancy in the release profiles is due to the functionality of the quaternary ammonium groups. As stated earlier, the Eudragit ${ }^{\circledR}$ RS-types are poorly permeable, therefore the diffusion of the drug out of the system is slower, leading to lower drug concentrations in solution. Similar results were obtained by Haznedar and Dortunç (2004) when the same microspheres were prepared by a conventional technique.

The preparation of polymer blends co-precipitated with acetazolamide can create tailor-made solutions with the desired drug release profile. By supercritical semi-continuous technique, three blends were precipitated in the presence of acetazolamide. The drug load was kept constant so that the influence of the blend composition upon the release rate could be evaluated (Fig. 11)

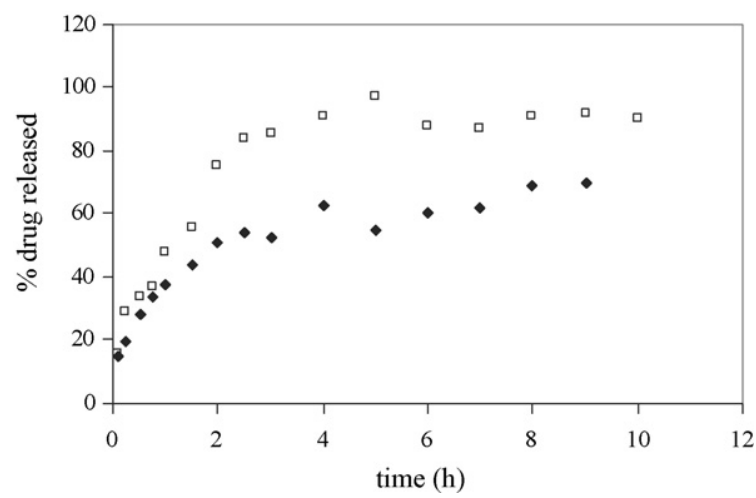

Fig. 10. Release profiles of the system Eudragit RS $100+$ acetazolamide (run \#2) (\) $\mathrm{pH} 1.2$ and $(\square) \mathrm{pH} 7.4$. 


\begin{tabular}{|l|c|c|c|}
\hline & $\begin{array}{c}\text { Eudragit } \\
\text { RS }\end{array}$ & $\begin{array}{c}\text { Eudragit } \\
\text { RL }\end{array}$ & Run\# \\
\hline$\diamond$ & 70 & 30 & 5 \\
\hline$*$ & 50 & 50 & 3 \\
\hline$\diamond$ & 30 & 70 & 4 \\
\hline
\end{tabular}

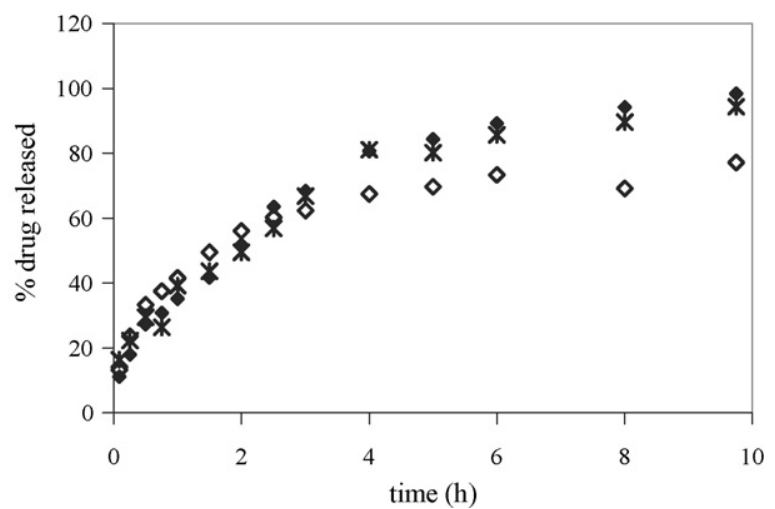

Fig. 11. Release profiles ( $\mathrm{pH}$ 1.2) of the systems described in the table, where the ratio of the two polymers is evidenced.

From the experiments performed with the polymers alone and the co-precipitation of polymer blends, we can conclude that it is possible to control the drug release from the particles produced if the ratio between the two polymers Eudragit RS and RL is varied. In Fig. 11 it is evidenced that an increase of the Eudragit RL percentage enhances the release of the drug in the aqueous media. The same behaviour was observed with PBS at $\mathrm{pH}$ 7.4.

The release profiles of particles produced by the batch operation were also studied in the two media. Samples were produced from same polymer blends of Eudragit RL and RS of 30:70, but with different conditions of temperature and overall concentration. Fig. 12 shows the behaviour at $\mathrm{pH}$ 1.2; comparison with the dissolution profile of pure acetazolamide, and the release profile of a semi-continuous sample (run \#5) is also provided. Among the batch samples, the agglomeration and the particle size of the powder obviously influenced the release rate of the drug. Particles prepared at higher pressure and temperature present large agglomerates and therefore, the drug release is slower. Like previously, the formulation with a polymer blends enable a sustained release of the drug into the aqueous media, but the ACTZ release was faster from the batch composite particles than from the semi-continuous ones.

To better understand the drug release mechanism of the systems prepared, the Fick's law of diffusion and the Power law

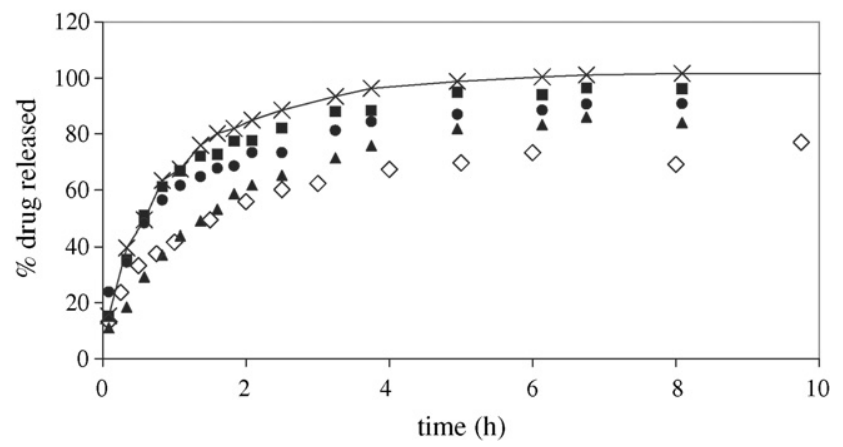

Fig. 12. Release profiles (pH 1.2) of the system acetazolamide + (Eudragit RS $100(70 \%)+$ Eudragit RL $100(30 \%))$ prepared by batch operation in various conditions (run \#6: ( $\mathbf{\Lambda})$, \#7: ( $)$, \#8: (ם) or obtained by semi-continuous operation (run \#5: $(\diamond))$; the dissolution profile of pure drug $(-\mathrm{x}-)$ is given for comparison. were applied to fit the release profiles (Siempmann and Peppas, 2001). The Fick's law is a special case of the Power law, when the release exponent is 0.5 . For the release range $M_{t} / M_{\infty}$ below $60 \%, M_{t} / M_{\infty}$ versus $t^{n}$, can be applied. Both the Fick's law and the Power law were found to fit well the release data of samples produced by the semi-continuous operation (Fig. 13, run \#5). The release exponents $n$ obtained were very closed to the values of $0.43-0.45$ that indicate a diffusion-controlled drug release in case of spherical or cylindric samples (Siempmann and Peppas, 2001). The Power law enables to determine the kinetic constants associated to the release of the drug from the produced systems. The kinetic constant for the release of acetazolamide from Eudragit RS was 0.26 and from Eudragit RL was 0.31. When a blend of the two polymers were co-precipitated with the drug, the values were within these two limits as expected. The composites produced by batch operation were found to exhibit a different behaviour. The fit of release profile with the Power law gave release exponents $n$ in the range of $0.56-0.61$, specially at $\mathrm{pH}$ 1.2 (Fig. 13, run \#6). However, the limits set to differentiate the mechanisms apply only if the particles are monodispersed, that is hardly the case of particles produced by the batch operation (see PSD profile reported in Fig. 7). Therefore, it is postulated that the release mechanism from batch-samples is due to both diffusion and swelling.

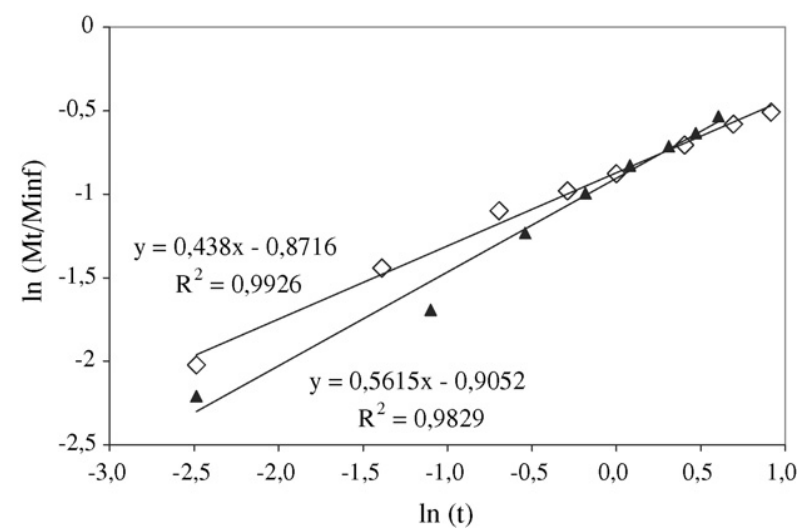

Fig. 13. Power law (pH 1.2) of the systems acetazolamide + (Eudragit RS 100 $(70 \%)+$ Eudragit RL $100(30 \%))$ prepared by batch (run \#6: (ム)) or semicontinuous operation (run \#5: $(\diamond)$ ). 


\section{Conclusions}

The preparation of acetazolamide composite particles for ophthalmic drug delivery was successfully performed with the use of $\mathrm{CO}_{2}$ as anti-solvent. Conditions that combined a production rate in the range of $70 \%$, a particle size distribution between 8 and $40 \mu \mathrm{m}$ and slowest release of the drug were obtained by the semi-continuous operation, carried out at $83 \mathrm{bar}, 37^{\circ} \mathrm{C}$ with $1 \mathrm{~mL} / \mathrm{min}$ liquid flow and $15 \mathrm{~mL} / \mathrm{min} \mathrm{CO}_{2}$ flow. Particles prepared by the batch operation had a larger mean size diameter and a larger size distribution than semi-continuous samples.

The rate of the release of the drug into aqueous media could be controlled to some extend by the preparation of these particles, either by using two versions of the anti-solvent technique, or by varying the ratio of two types of Eudragit as polymer blend. Formulations enriched in Eudragit RS lead to a slower release of acetazolamide than those enriched in Eudragit RL, whereas samples processed by batch exhibit faster profiles than those processed by the semi-continuous mode. The compliance of the release profiles of semi-continuous samples with the Power law suggests that the drug release is controlled by diffusion mechanism, and the derived kinetic constant is increasing as the content of Eudragit RL in the polymer blend increases. For samples produced by batch operation, their faster release despite a larger particle size, their compliance with the Power law, and the increase of the rate with a decrease of particle size, suggest that the polymer swelling intervenes in the release transport although diffusion is still predominant.

Therefore, various controlled release systems for the delivery of acetazolamide was successfully prepared using a supercritical anti-solvent process.

\section{Acknowledgements}

Ana Rita C. Duarte is grateful for financial support from FCT through SFRH/BD/10780/2002 grant. Authors gratefully acknowledge the support of French-Portuguese PAUILF 2005 program.

\section{References}

http://www.roehm.de/en/pharmapolymers?content=/en/pharmapolymers/ formulation/sustaine.

Brannon-Peppas, L., 1997. Polymers in controlled drug delivery. Med. Plast. Biomater. (available online).
Chattopadhyay, P., Gupta, R.B., 2002. Supercritical CO2 based production of magnetically responsive micro- and nanoparticles for drug targeting. Ind. Eng. Chem. Res. 41, 6049-6058.

CPMP/ICH/283/95, 1997. ICH guideline Q3C, Impurities Residual Solvents.

De Gioannis, B., Patrick, J., Subra, P., 2004. Morphology and growth control of griseofulvin recrystallised by compressed carbon dioxide as antisolvent. J. Crystal. Growth 262, 519-526.

Haznedar, S., Dortunç, B., 2004. Preparation and in vitro evaluation of Eudragit microspheres containing acetazolamide. Int. J. Pharm. 269, 131-140.

Heller, J., 1996. Drug delivery systems. In: Ratner, B.D., Hoffmann, A.S., Schoen, F.J., Lemons, J.E. (Eds.),Drug delivery systems. Biomaterials Science, An Introduction to Materials in Medicine. Academic Press, London, UK, pp. 346-356.

Jung, J., Perrut, M., 2001. Particle design using supercritical fluids: literature and patent survey. J. Supercrit. Fluids 20, 179-219.

Juppo, A.M., Boissier, C., Khoo, C., 2003. Evaluation of solid dispersion particles prepared with SEDS. Int. J. Pharm. 250, 385-401.

Kaur, I.P., Smitha, R., Aggarwal, D., Kapil, M., 2002. Acetazolamide: future perspectives in topical glaucoma therapeutics. Int. J. Pharm. 248, 1-14.

Pignatello, R., et al., 2003. Flurbiprofen-loaded acrylate polymer nanosuspensions for ophthalmic application. Biomaterials 23, 3247-3255.

Reverchon, E., 2002. Micro and nano particles produced by supercritical fluid assisted techniques: present status and perspectives.Micro and nano particles produced by supercritical fluid assisted techniques: present status and perspectives. In: Proceedings of the 4th International Symposium on High Pressure Technology and Chemical Engineering, Venice, Italy.

Reverchon, E., Adami, R., 2005. Nanomaterials and supercritical fluids. J. Supercrit. Fluids (available on line).

Siempmann, J., Peppas, N.A., 2001. Modelling of drug release from delivery systems based on hydroxypropyl methylcellulose (HPMC). Adv. Drug Del. Rev. 48, 139-157.

Subra, P., Jestin, P., 1999. Powders elaboration in supercritical media: comparison with conventional routes. Powder Technol. 103, 2-9.

Vega-González, A., Domingo, C., Elvira, C., Subra, P., 2004. Precipitation of PMMA/PCL blends using supercritical carbon dioxide. J. Appl. Polymer Sci. 91, 2422-2426.

Vega-González, A., Duarte, A.R.C., Subra-Paternault, P., Duarte, C.M.M., 2005. Preparation of acetazolamide drug delivery systems using a supercritical antisolvent process.Preparation of acetazolamide drug delivery systems using a supercritical antisolvent process. In: Proceedings of the 10th European Meeting on Supercritical Fluids, Colmar, France, p. 39.

Vemavarapu, C., Mollan, M.J., Lodaya, M., Needhamb, T.E., 2005. Design and process aspects of laboratory scale SCF particle formation systems. Int. J. Pharm. 292, 1-16.

Verreck, G., Decorte, A., Li, H., Tomasko, D., Arien, A., Peeters, J., Rombaut, P., Van den Mooter, G., Brewster, M.E., in press. The effect of pressurized carbon dioxide as a plasticizer and foaming agent on the hot melt extrusion process and extrudate properties of pharmaceutical biopolymers. J. Supercrit. Fluids.

Wang, Y., Dave, R.N., Pfeffer, R., 2004. Polymer coating/encapsulation of nanoparticles using a supercritical anti-solvent process. J. Supercrit. Fluids 28, 85-99. 